J. Perinat. Med. $11(1983) 204$

\section{Prenatal diagnosis and treatment of intrauterine growth retardation}

\author{
T. Kaneoka, S. Taguchi, H. Shimizu, K. Shirakawa
}

Department of Obstetrics and Gynecology, Fukuoka University

School of Medicine, Fukuoka, 814-01, Japan

(Director: Prof. Koichi Shirakawa)
Intrauterine growth retardation (IUGR) is one of the most important fetal complications in perinatology. With the recent advances in ultrasonic measurements, the early recognition of fetal maldevelopment has become possible.

In a prospective study of pregnancies with a fetal body weight ultrsonographically determined to be less than the 10th percentile of population, prenatal treatment consisting of daytime bedrest, high protein diet and oral administration of allylestrenol was assessed.

\section{Material and Methods}

A total of 30 pregnancies was investigated. In these pregnancies, the intrauterine gestational age was confirmed in the frist trimester of pregnancy by routine ultrasonic measurements of crown-rump length (CRL), using the standard of ROBINSON [5]. Both biparietal diameter (BPD) and abdominal circumference (AC) were measured in the third trimester. An ultrasonograph (Aloka, SSD-256) with a $3.5 \mathrm{MHz}$ transducer was used in both the first and third trimester measurements. The ultrasound tissue velocity used was 1,540 meters per second. CRL was measured with electronic calipers when the fetal echo complex was shown at its maximum length. BPD scan was obtained when the cranium became oval at an angle so that the clear midline structure and the lateral ventricles could be observed. The distance between the frontal lateral margin to the posterior internal margin of the cranium was measured with electronic calipers. A photograph was made when the umbilical vein could be observed in a transverse scan at the right angle

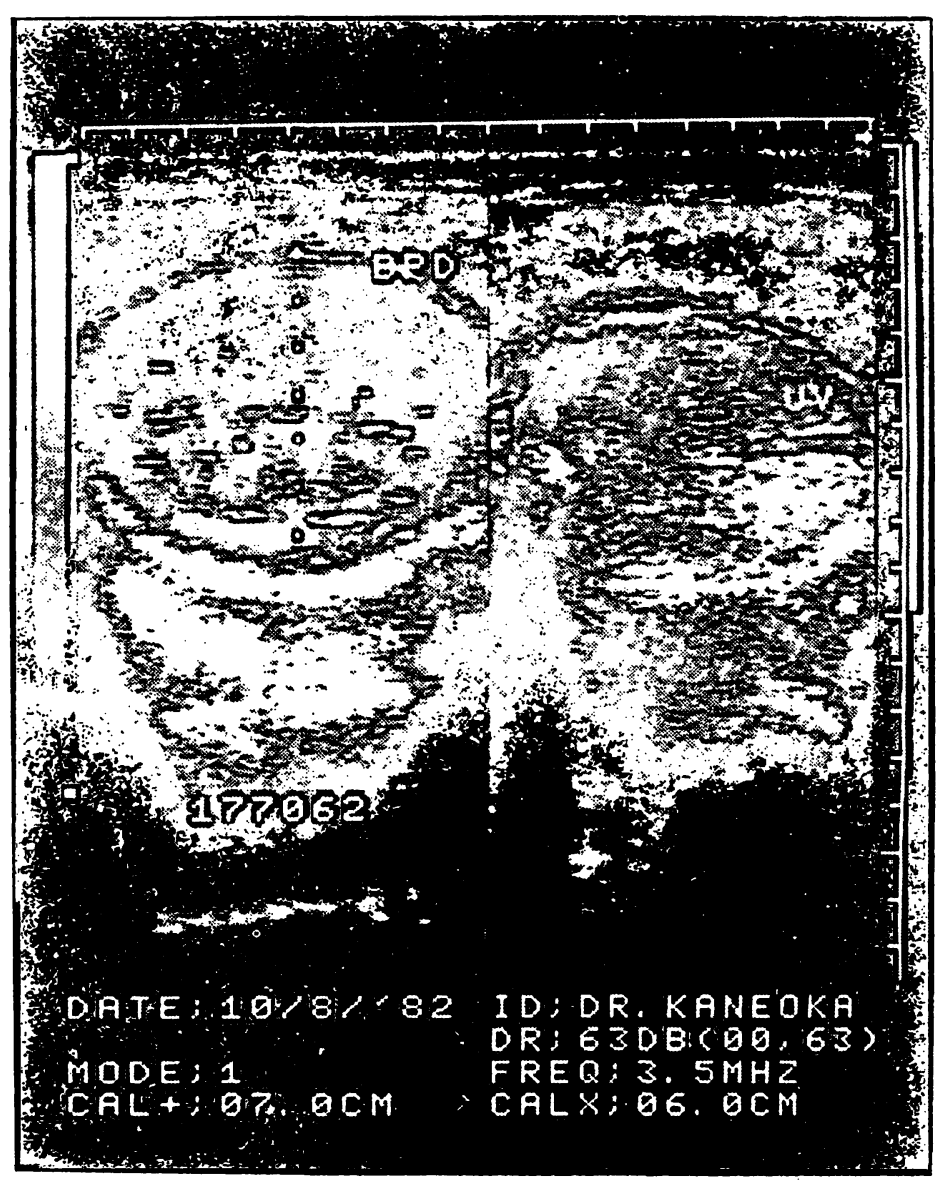

Fig. 1. Biparietal diameter (BPD) and abdominal circumference $(A C)$. 
to the long axis of the fetus, and the $\mathrm{AC}$ was measured by a map reader (Uchida) (Fig. 1). Then, the fetal weight was estimated from the $B P D$ and AC measurements in accordance with the method of WARSOF et al. [14] and SHEPARD et al. [8], using an equation of $\log _{10}$ (body weight) $=-1.599+0.144(\mathrm{BPD})+0.032(\mathrm{AC})-0.111$ $\left(\mathrm{BPD}^{2} \times \mathrm{AC}\right) / 1,000$ (equation $\left.\mathrm{I}\right)$ and an equation of $\log _{10}$ (body weight) $=-1.7492+0.166$ (BPD) $+0.046(\mathrm{AC})-2.646(\mathrm{AC} \times \mathrm{BPD}) / 1,000$ (equation II). In those 30 pregnancies, each the estimated fetal body weight was less than 10th percentile of the intrauterine growth curve of NISHIDA [4] for the Japanese at more than two ultrasonic measurements separated by two weeks. Maternal biochemical determinations were also performed on these pregnancies. The plasma unconjugated estriol value was measured with a CEA-IRE-Sorin RIA kit. Urinary estriol was determined on an 8-hour pooled specimen collected at night with a Teizo colorimetric kit and converted into that of a 24-hour urine. Plasma human placental lactogen (HPL) was assayed with a Kyorin EIA kit. Plasma unconjugated progesterone value was measured with a Daiichi RIA kit. Serum heat-stable alkaline phosphatase (HSAP) and leucine amino-peptidase (LAP) were determined colorimetrically. The patients were treated with daytime bedrest, high protein diet $(2 \mathrm{~g} / \mathrm{kg} /$ day $)$ and oral administration of allylestrenol (GESTANON ${ }^{\circledR}$, NIPPON ORGANON) in a dose of $30 \mathrm{mg} /$ day until the time of delivery. Both ultrasonic and biochemical measurements were repeated weekly. For statistical analysis, Student's t-test was applied.

\section{Results}

The calculated values of the various measurements obtained prior to and following treatments were as follows.

\subsection{Estimated fetal body weight}

As shown in Tab. I, the initial gestational age of the 30 patients who were diagnosed as IUGR after at least two measurements taken two weeks apart were an average of $32.9 \pm$ S.D. 2.4 weeks, and the estimated average fetal body weight was $1,362 \pm 316 \mathrm{~g}$ by equation $\mathrm{I}$ and $1,430 \pm 337 \mathrm{~g}$ by equation II. In the final assessment at $39.2 \pm 1.8$ weeks' gestation, the estimated average fetal body weight had increased to $2,678 \pm 458 \mathrm{~g}$ by equation I and to $2,787 \pm 477 \mathrm{~g}$ by equation II. Therefore, the average weekly gain of estimated fetal body weight was $223 \pm 83 \mathrm{~g}$ by equation I and $219 \pm 64 \mathrm{~g}$ by equation II, both of which were statistically significantly $(\mathrm{p}<0.01)$ larger than the standard weekly increase at the 50th percentile level of NISHIDA's intrauterine growth curve [4], $171 \pm 42 \mathrm{~g}$, confirming the efficacy of prenatal threapy. Intrauterine weight chart of each case is illustrated on Fig. 2.

\subsection{Estimated fetal body weight and actual birth weight}

As stated above, the final estimated fetal body weight was $2,678 \pm 458 \mathrm{~g}$ by equation I and $2,787 \pm 477 \mathrm{~g}$ by equation II, on average, at $39.2 \pm 1.8$ weeks' gestation, and the actual birth weight was an average of $2,685 \pm 479 \mathrm{~g}$ at $39.5 \pm 1.7$ weeks' gestation. Significant correlations ( $r=0.94$ on both equations) between the estimated fetal body weight and the actual birth weight were found, and it was confirmed that these estimating methods were useful in the prenatal diagnosis of IUGR. Standard deviations in birth weight predictions were $178 \mathrm{~g}$ in equation I and $259 \mathrm{~g}$ in equation II. With respect to the birth weight, $14(46.6 \%)$ showed a lower weight than 10th percentile of NiSHIDA's growth curve [4]. The average placental weight of these cases was $490 \pm 95 \mathrm{~g}$. The fetal/placental weight ratio was $5.52 \pm 0.64$ on average, which was somewhat lower than that of normal pregnancy (usually, between 5.9 and 6.3). Fetal distress was not encountered in any of these cases during the course of delivery. All neonates had a one-minute APGAR Score of more than 8.

\section{3 $\mathrm{BPD}$ and $\mathrm{AC}$ measurements}

The BPD and AC measurements taken at $32.9 \pm 2.4$ weeks' gestation were $76 \pm 6 \mathrm{~mm}$ and $25 \pm 2 \mathrm{~cm}$, respectively, and those at $39.2 \pm 1.8$ weeks' gestation were $87 \pm 4 \mathrm{~mm}$ and $33 \pm 2 \mathrm{~cm}$, respectively. On the basis of SABBAGHA's standard [6], the BPD was below 10th percentile in 23 cases $(76.7 \%)$ at the initial measurement, and in $22(73.3 \%)$ at the 


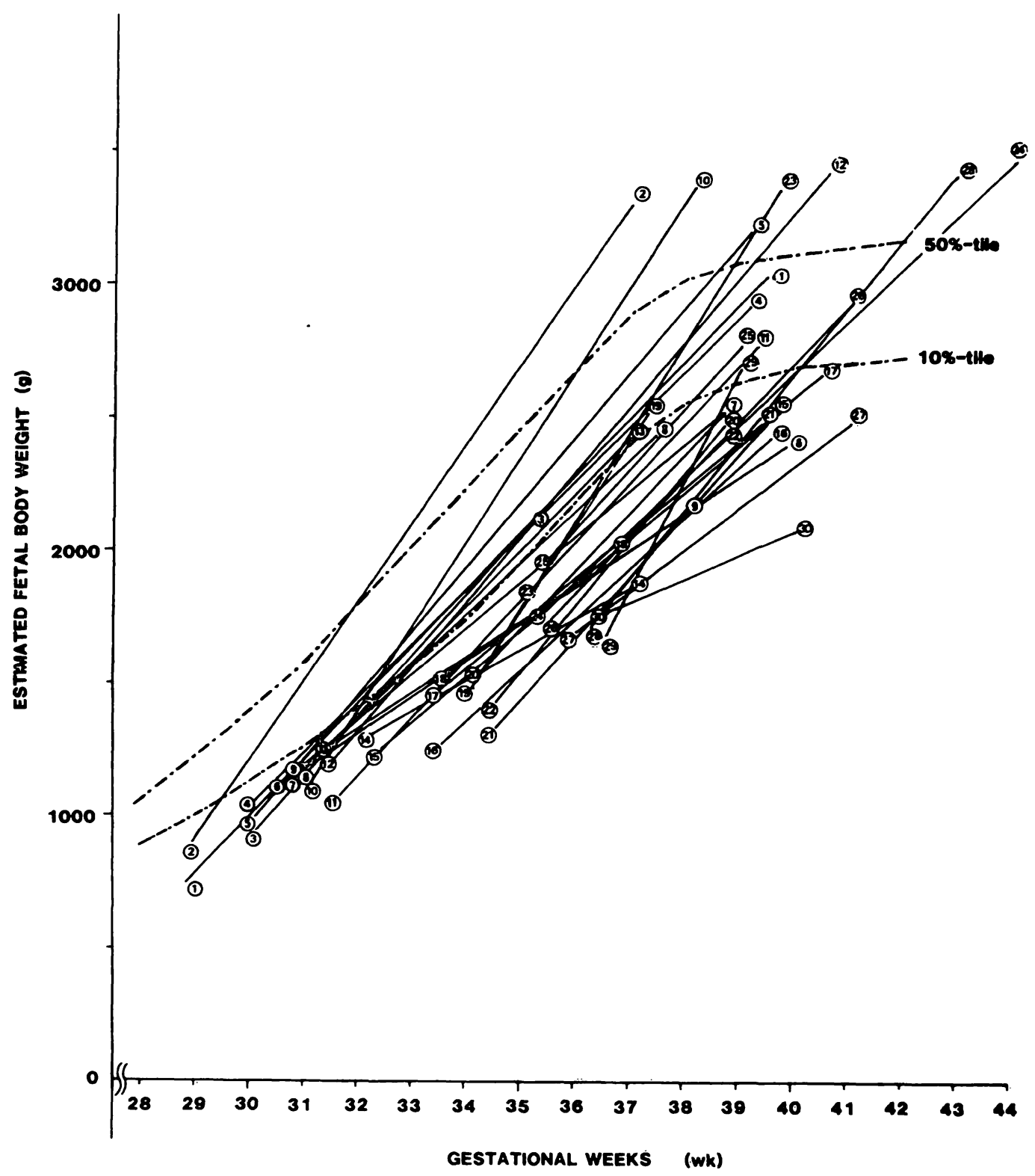

Fig. 2. Intrauterine weight chart. Equation I was used in calculation.

final measurement. Similarly, on the basis of TAMURA's standard [11], the AC was below the 10 th percentile in $30(100 \%)$ at the initial measurement, and in $21(70.0 \%)$ at the final measurement. The average weekly increase of BPD was $1.8 \pm 0.6 \mathrm{~mm}$, which was insignificant to the standard gain of $1.6 \pm 0.3 \mathrm{~mm}$ according to SABBAGHA's values [6] at 50th percentile level. The average weekly increase of $A C$ was $1.3 \pm 0.4 \mathrm{~cm}$, which was a statistically significant $(p<0.01)$ increase over the standard gain of $1.1 \pm 0.2 \mathrm{~cm}$ according to TAMURA's value [11] at 50 th percentile level.

\subsection{Maternal biochemical values}

Tab. II demonstrates the maternal plasma unconjugated estriol, urinary estriol, plasma HPL, plasma unconjugated progesterone, serum HSAP and serum LAP concentration values at $32.9 \pm 2.4$ weeks' gestation and the values following three weeks of prenatal treatment. The initial plasma unconugated estriol value was $2.9 \pm 2.2 \mathrm{ng} / \mathrm{ml}$ on average, and in 20 patients $(66.7 \%)$ the value was abnormally low on the basis of TULCHINSKY's standard [12]. Three weeks after treatment it was $5.2 \pm 3.5 \mathrm{ng} / \mathrm{ml}$ which was a statistically significant $(p<0.01)$ increase, but 15 (50.0\%) 
Tah. I. Fetal biparictal diameter (BPD), abdominal circumference (AC) and estimated body weight before and after prenatal treatment, actual birth weight and placental weight.

\begin{tabular}{|c|c|c|c|c|c|c|c|c|c|c|c|}
\hline \multirow{3}{*}{ No. } & \multicolumn{5}{|c|}{ Initial Measurements } & \multicolumn{6}{|c|}{ Final Mcasurements } \\
\hline & \multirow{2}{*}{$\begin{array}{l}\text { Ciss. } \\
\text { Whs. } \\
W+D\end{array}$} & \multirow{2}{*}{ BPI } & \multirow{2}{*}{$\begin{array}{l}\text { AC: } \\
\mathrm{cm}\end{array}$} & \multicolumn{2}{|c|}{$\begin{array}{l}\text { Estimated } \\
\text { Body Weight } \\
\text { Eq.1 Eq.II }\end{array}$} & \multirow{2}{*}{$\begin{array}{l}\text { Ges. } \\
\text { Wks. } \\
W+D\end{array}$} & \multirow{2}{*}{$\begin{array}{l}\text { BPD } \\
\mathrm{mm}\end{array}$} & \multirow{2}{*}{$\begin{array}{l}\mathrm{AC} \\
\mathrm{cm}\end{array}$} & \multirow{2}{*}{\multicolumn{2}{|c|}{$\begin{array}{l}\text { Estimated } \\
\text { Body Weight } \\
\text { Eq.I } \\
\begin{array}{ll}\text { B } & \text { Eq. II }\end{array}\end{array}$}} & \multirow{2}{*}{$\begin{array}{l}\text { Actual } \\
\text { Birtl } \\
\text { Weight } \\
\text { g }\end{array}$} \\
\hline & & & & g & 8 & & & & & & \\
\hline $\begin{array}{l}1 . \\
2 . \\
3 . \\
4 . \\
5 .\end{array}$ & $\begin{array}{l}28+6 \\
29+0 \\
30+1 \\
30+1 \\
30+1\end{array}$ & $\begin{array}{l}62^{*} \\
69^{\circ} \\
66^{\circ} \\
68^{*} \\
73^{*}\end{array}$ & $\begin{array}{l}21^{*} \\
21^{*} \\
23^{*} \\
24^{*} \\
21^{*}\end{array}$ & $\begin{array}{r}751^{*} \\
902^{*} \\
946^{*} \\
1059^{*} \\
999^{*}\end{array}$ & $\begin{array}{l}792^{*} \\
952^{*} \\
1056^{*} \\
1126^{*} \\
1054^{*}\end{array}$ & $\begin{array}{l}39+3 \\
37+0 \\
35+1 \\
39+1 \\
39+1\end{array}$ & $\begin{array}{l}87^{*} \\
92 \\
74^{*} \\
88^{*} \\
90\end{array}$ & $\begin{array}{l}35 \\
35 \\
33 \\
34 \\
35\end{array}$ & $\begin{array}{l}3018 \\
3288 \\
2098^{*} \\
2910 \\
3179\end{array}$ & $\begin{array}{l}3157 \\
3350 \\
2137^{*} \\
3047 \\
3237\end{array}$ & $\begin{array}{l}2911 \\
2890 \\
2060^{*} \\
2952 \\
3141\end{array}$ \\
\hline $\begin{array}{c}6 . \\
7 . \\
8 . \\
9 . \\
10 .\end{array}$ & $\begin{array}{l}30+4 \\
30+5 \\
31+0 \\
31+0 \\
31+1\end{array}$ & $\begin{array}{l}73^{*} \\
76 \\
75 \\
73 * \\
73^{*}\end{array}$ & $\begin{array}{l}23^{*} \\
22^{*} \\
23^{*} \\
24^{*} \\
23^{*}\end{array}$ & $\begin{array}{l}1127^{\circ} \\
1143^{\circ} \\
1184^{\circ} \\
1197^{\circ} \\
1127^{\circ}\end{array}$ & $\begin{array}{l}1192^{\circ} \\
1178^{\circ} \\
1221^{\circ} \\
1229^{\circ} \\
1192^{*}\end{array}$ & $\begin{array}{l}39+6 \\
38+5 \\
37+3 \\
38+0 \\
38+1\end{array}$ & $\begin{array}{l}86^{*} \\
86^{*} \\
87^{*} \\
86^{*} \\
90^{\circ}\end{array}$ & $\begin{array}{l}31^{*} \\
32^{*} \\
31^{*} \\
29^{*} \\
36\end{array}$ & $\begin{array}{l}2381^{*} \\
2515^{*} \\
2428 \\
2134^{*} \\
3352\end{array}$ & $\begin{array}{l}2457^{*} \\
2643^{*} \\
2554 \\
2251^{*} \\
3495\end{array}$ & $\begin{array}{l}2447^{*} \\
2633^{*} \\
2833 \\
2150^{*} \\
3324\end{array}$ \\
\hline $\begin{array}{l}11 . \\
12 . \\
13 . \\
14 . \\
15\end{array}$ & $\begin{array}{l}31+4 \\
31+4 \\
31+4 \\
32+2 \\
32+3\end{array}$ & $\begin{array}{l}71^{*} \\
74^{*} \\
73^{*} \\
73^{*} \\
75^{*}\end{array}$ & $\begin{array}{l}23^{*} \\
24^{*} \\
25^{*} \\
25^{*} \\
24^{*}\end{array}$ & $\begin{array}{l}1073^{*} \\
1226^{*} \\
1271^{*} \\
1310^{*} \\
1256^{*}\end{array}$ & $\begin{array}{l}1106^{*} \\
1267^{*} \\
1307^{*} \\
1348^{*} \\
1297^{*}\end{array}$ & $\begin{array}{l}39+2 \\
40+4 \\
37+3 \\
37+0 \\
39+5\end{array}$ & $\begin{array}{l}88^{*} \\
91 \\
84 * \\
82^{*} \\
83^{*}\end{array}$ & $\begin{array}{l}33^{*} \\
36 \\
32^{*} \\
28^{*} \\
33^{*}\end{array}$ & $\begin{array}{l}2757 \\
3407 \\
2420^{*} \\
1857^{*} \\
2511^{*}\end{array}$ & $\begin{array}{l}2892 \\
3552 \\
2546 \\
1906 * \\
2641\end{array}$ & $\begin{array}{l}2930 \\
3310 \\
2407^{*} \\
1651 * \\
2545^{*}\end{array}$ \\
\hline $\begin{array}{l}16 . \\
17 . \\
18 . \\
19 . \\
20 .\end{array}$ & $\begin{array}{l}33+4 \\
33+5 \\
33+5 \\
34+1 \\
34+2\end{array}$ & $\begin{array}{l}73^{*} \\
80 \\
76^{*} \\
82 \\
79^{*}\end{array}$ & $\begin{array}{l}25^{*} \\
25^{*} \\
27^{*} \\
24^{*} \\
26^{*}\end{array}$ & $\begin{array}{l}1271^{*} \\
1479^{*} \\
1535^{*} \\
1481^{*} \\
1550^{*}\end{array}$ & $\begin{array}{l}1317^{*} \\
1547^{*} \\
1592^{*} \\
1531^{*} \\
1603^{*}\end{array}$ & $\begin{array}{l}39+4 \\
40+3 \\
36+5 \\
37+2 \\
38+5\end{array}$ & $\begin{array}{l}84^{*} \\
86^{*} \\
80^{*} \\
90 \\
86^{*}\end{array}$ & $\begin{array}{l}32^{*} \\
33^{*} \\
30^{*} \\
30^{*} \\
32^{*}\end{array}$ & $\begin{array}{l}2420^{*} \\
2657 \\
1994^{*} \\
2504^{*} \\
2515^{*}\end{array}$ & $\begin{array}{l}2546^{*} \\
2789 \\
2107^{*} \\
2572 \\
2573^{*}\end{array}$ & $\begin{array}{l}2379 * \\
2735 \\
1945^{*} \\
2463 \\
2520^{*}\end{array}$ \\
\hline $\begin{array}{l}21 . \\
22 . \\
23 . \\
24 . \\
25\end{array}$ & $\begin{array}{l}34+4 \\
34+4 \\
35+2 \\
35+3 \\
35+4\end{array}$ & $\begin{array}{l}75^{*} \\
78^{*} \\
85 \\
85 \\
85\end{array}$ & $\begin{array}{l}25^{*} \\
25^{*} \\
27^{*} \\
26^{*} \\
28^{*}\end{array}$ & $\begin{array}{l}1333^{*} \\
1430^{*} \\
1872^{*} \\
1772^{*} \\
1978^{*}\end{array}$ & $\begin{array}{l}1379^{*} \\
1477^{*} \\
1979 * \\
1875^{*} \\
2089 *\end{array}$ & $\begin{array}{l}39+3 \\
38+5 \\
39+5 \\
44+0 \\
39+0\end{array}$ & $\begin{array}{l}88^{*} \\
85^{*} \\
93 \\
95 \\
88^{*}\end{array}$ & $\begin{array}{l}31^{*} \\
32^{*} \\
35 \\
35 \\
33^{*}\end{array}$ & $\begin{array}{l}2475^{*} \\
2468^{*} \\
3342 \\
3454 \\
2757\end{array}$ & $\begin{array}{l}2537 * \\
2594^{*} \\
3494 \\
3614 \\
2817\end{array}$ & $\begin{array}{l}2446 * \\
2290^{*} \\
3645 \\
3216 \\
2895\end{array}$ \\
\hline $\begin{array}{l}26 . \\
27 . \\
28 . \\
29 . \\
30 .\end{array}$ & $\begin{array}{l}35+4 \\
36+0 \\
36+2 \\
36+4 \\
36+4\end{array}$ & $\begin{array}{l}79 * \\
83^{*} \\
81^{*} \\
80^{*} \\
85^{*}\end{array}$ & $\begin{array}{l}28^{*} \\
26^{*} \\
27^{*} \\
27^{*} \\
26^{*}\end{array}$ & $\begin{array}{l}1740^{*} \\
1695^{*} \\
1716^{*} \\
1679^{*} \\
1772^{*}\end{array}$ & $\begin{array}{l}1840^{*} \\
1875^{*} \\
1814^{*} \\
1775^{*} \\
1723^{*}\end{array}$ & $\begin{array}{l}41+0 \\
41+0 \\
42+6 \\
39+0 \\
40+0\end{array}$ & $\begin{array}{l}88^{*} \\
88^{*} \\
94 \\
86^{*} \\
87^{*}\end{array}$ & $\begin{array}{l}34^{*} \\
31^{*} \\
35^{*} \\
33^{*} \\
28^{*}\end{array}$ & $\begin{array}{l}2910 \\
2475^{*} \\
3398 \\
2675 \\
2063^{*}\end{array}$ & $\begin{array}{l}3047 \\
2604^{*} \\
3553 \\
2715 \\
2122^{*}\end{array}$ & $\begin{array}{l}3207 \\
2685^{*} \\
3269 \\
2820 \\
1890^{*}\end{array}$ \\
\hline $\begin{array}{l}\text { Mean } \\
\text { S.D. }\end{array}$ & $\begin{array}{r}32.9 \\
2.4\end{array}$ & $\begin{array}{r}76 \\
6\end{array}$ & $\begin{array}{r}25 \\
2\end{array}$ & $\begin{array}{r}1362 \\
316\end{array}$ & $\begin{array}{r}1430 \\
337\end{array}$ & $\begin{array}{r}39.2 \\
1.8\end{array}$ & $\begin{array}{r}87 \\
4\end{array}$ & $\begin{array}{r}33 \\
2\end{array}$ & $\begin{array}{r}2678 \\
458\end{array}$ & $\begin{array}{r}2787 \\
477\end{array}$ & $\begin{array}{r}2685 \\
479\end{array}$ \\
\hline
\end{tabular}

Note) ${ }^{*}$ less than 10 th percentile. UPI: utero-placental insufficiency. UTI: urinary tract infection. np: nullipara.

still showed abnormally low values. Likewise, the urinary estriol value at the initial measurement was an average of $13.3 \pm 6.5 \mathrm{mg} /$ day, and 8 cases (26.7\%) had abnormally low values on the basis of BEISCHER's standard [2]. Three weeks after treatment it increased to $23.4 \pm 10.2 \mathrm{mg} /$ day which was a statistically significant $(\mathrm{p}<0.01)$ increase, but only two $(6.7 \%)$ of the patients had abnormally low values. The plasma HPL value was an average of $4.8 \pm 1.2 \mu \mathrm{g} / \mathrm{ml}$ at the initial measure- ment, and on the basis of VARMA's standard [13] $6(20.0 \%)$ showed an abnormally low value. Three weeks after treatment the average value was $5.9 \pm 1.6 \mu \mathrm{g} / \mathrm{ml}$, which was statistically significant $(p<0.01)$ increase in value, but $3(10.0 \%)$ had an abnormally low value. Initially, the plasma unconjugated progesterone value was $98 \pm 26 \mathrm{ng} / \mathrm{ml}$, but after three weeks' treatment it increased to $121 \pm 35 \mathrm{ng} / \mathrm{ml}$, which was statistically significant $(p<0.01)$ increase. The serum HSAP and LAP 
Tab. I.

Continued.

\begin{tabular}{|c|c|c|c|c|c|c|c|c|c|}
\hline \multirow{3}{*}{ No. } & \multicolumn{4}{|c|}{ Average Weekly Gain } & \multicolumn{5}{|c|}{ Standard Gain } \\
\hline & \multirow{2}{*}{$\begin{array}{l}\text { BPD } \\
\mathrm{mm}\end{array}$} & \multirow{2}{*}{$\begin{array}{l}\mathrm{AC} \\
\mathrm{cm}\end{array}$} & \multicolumn{2}{|c|}{$\begin{array}{l}\text { Estimated } \\
\text { Body Weight } \\
\text { Eq.I } \quad \text { Eq.II }\end{array}$} & \multirow{2}{*}{$\begin{array}{l}\text { BPD } \\
\mathrm{mm}\end{array}$} & \multirow{2}{*}{$\begin{array}{l}\mathrm{AC} \\
\mathrm{cm}\end{array}$} & $\begin{array}{l}\text { Body } \\
\text { Weight }\end{array}$ & \multirow{2}{*}{$\begin{array}{l}\text { Placental } \\
\text { Weight } \\
\text { g }\end{array}$} & \multirow[t]{2}{*}{ Complication } \\
\hline & & & $\mathbf{g}$ & $\mathbf{g}$ & & & $\mathbf{g}$ & & \\
\hline $\begin{array}{l}1 . \\
2 . \\
3 . \\
4 . \\
5 .\end{array}$ & $\begin{array}{l}2.4 \\
2.9 \\
1.6 \\
2.2 \\
1.9\end{array}$ & $\begin{array}{l}1.4 \\
1.8 \\
2.0 \\
1.1 \\
1.6\end{array}$ & $\begin{array}{l}214 \\
298 \\
230 \\
206 \\
242\end{array}$ & $\begin{array}{l}206 \\
300 \\
216 \\
213 \\
243\end{array}$ & $\begin{array}{l}1.9 \\
2.1 \\
2.0 \\
1.8 \\
1.8\end{array}$ & $\begin{array}{l}0.9 \\
1.0 \\
1.0 \\
0.9 \\
0.9\end{array}$ & $\begin{array}{l}183 \\
208 \\
212 \\
187 \\
187\end{array}$ & $\begin{array}{l}502 \\
490 \\
410 \\
258 \\
462\end{array}$ & $\begin{array}{l}\text { hepatitis B } \\
\text { pre-eclampsia } \\
\text { elderly n.p. } \\
\text { elderly n.p. } \\
\text { pre-eclampsia }\end{array}$ \\
\hline $\begin{array}{r}6 . \\
7 . \\
8 . \\
9 . \\
10 .\end{array}$ & $\begin{array}{l}1.4 \\
1.3 \\
1.9 \\
1.9 \\
2.4\end{array}$ & $\begin{array}{l}0.9 \\
1.3 \\
1.3 \\
0.7 \\
1.9\end{array}$ & $\begin{array}{l}135 \\
172 \\
193 \\
134 \\
318\end{array}$ & $\begin{array}{l}136 \\
183 \\
207 \\
146 \\
329\end{array}$ & $\begin{array}{l}1.7 \\
1.0 \\
2.0 \\
1.9 \\
1.1\end{array}$ & $\begin{array}{l}0.9 \\
1.8 \\
1.1 \\
1.1 \\
1.9\end{array}$ & $\begin{array}{l}171 \\
187 \\
206 \\
204 \\
205\end{array}$ & $\begin{array}{l}538 \\
470 \\
497 \\
380 \\
578\end{array}$ & rheumatism \\
\hline $\begin{array}{l}11 \\
12 . \\
13 \\
14 . \\
15\end{array}$ & $\begin{array}{l}2.2 \\
1.9 \\
1.9 \\
1.9 \\
1.1\end{array}$ & $\begin{array}{l}1.3 \\
1.3 \\
1.0 \\
0.6 \\
1.2\end{array}$ & $\begin{array}{l}218 \\
242 \\
187 \\
124 \\
172\end{array}$ & $\begin{array}{l}231 \\
254 \\
211 \\
118 \\
184\end{array}$ & $\begin{array}{l}1.8 \\
0.9 \\
2.0 \\
1.8 \\
1.5\end{array}$ & $\begin{array}{l}1.0 \\
1.7 \\
1.1 \\
1.1 \\
0.9\end{array}$ & $\begin{array}{l}187 \\
169 \\
218 \\
215 \\
181\end{array}$ & $\begin{array}{l}630 \\
605 \\
366 \\
280 \\
380\end{array}$ & $\begin{array}{l}\text { pl. previa } \\
\text { U. .̃.I. } \\
\text { heart desease } \\
\text {-U.P.I. }\end{array}$ \\
\hline $\begin{array}{l}16 . \\
17 . \\
18 . \\
19 . \\
20 . \\
21 . \\
22 . \\
23 . \\
24 . \\
25 .\end{array}$ & $\begin{array}{l}1.8 \\
0.9 \\
1.3 \\
2.6 \\
1.6 \\
2.7 \\
1.7 \\
1.8 \\
1.2 \\
0.8\end{array}$ & $\begin{array}{l}1.2 \\
1.2 \\
1.0 \\
1.9 \\
1.4 \\
1.2 \\
1.7 \\
1.8 \\
1.0 \\
1.5\end{array}$ & $\begin{array}{l}192 \\
159 \\
153 \\
326 \\
218 \\
235 \\
251 \\
332 \\
196 \\
227\end{array}$ & $\begin{array}{l}205 \\
185 \\
172 \\
331 \\
219 \\
238 \\
270 \\
341 \\
203 \\
212\end{array}$ & $\begin{array}{l}1.5 \\
1.3 \\
1.7 \\
1.7 \\
1.4 \\
1.4 \\
1.4 \\
1.4 \\
1.4 \\
0.9\end{array}$ & $\begin{array}{l}1.2 \\
1.0 \\
1.4 \\
1.4 \\
1.2 \\
1.2 \\
1.2 \\
0.8 \\
0.8 \\
1.5\end{array}$ & $\begin{array}{l}178 \\
156 \\
217 \\
218 \\
194 \\
168 \\
194 \\
130 \\
101 \\
155\end{array}$ & $\begin{array}{l}430 \\
446 \\
370 \\
495 \\
500 \\
590 \\
460 \\
754 \\
560 \\
500\end{array}$ & $\begin{array}{l}\text { asthma } \\
\text { pre-eclampsia } \\
\text { U.T.I. } \\
\text { pl.previa } \\
\text { asthma } \\
\text { pre eclampsia }\end{array}$ \\
\hline $\begin{array}{l}26 . \\
27 . \\
28 . \\
29 . \\
30 .\end{array}$ & $\begin{array}{l}1.7 \\
1.0 \\
2.0 \\
1.8 \\
0.6\end{array}$ & $\begin{array}{l}1.1 \\
1.0 \\
1.2 \\
1.8 \\
0.6\end{array}$ & $\begin{array}{r}215 \\
156 \\
256 \\
297 \\
85\end{array}$ & $\begin{array}{r}217 \\
146 \\
265 \\
307 \\
72\end{array}$ & $\begin{array}{l}1.4 \\
1.3 \\
1.3 \\
1.3 \\
1.3\end{array}$ & $\begin{array}{l}0.8 \\
0.8 \\
0.8 \\
0.7 \\
0.7\end{array}$ & $\begin{array}{r}113 \\
93 \\
82 \\
109 \\
106\end{array}$ & $\begin{array}{l}540 \\
555 \\
512 \\
505 \\
370\end{array}$ & $\begin{array}{l}\text { elderly n.p. } \\
\text { U.P.I. }\end{array}$ \\
\hline $\begin{array}{l}\text { Mean } \\
\text { S.D. }\end{array}$ & $\begin{array}{l}1.8 \\
0.6\end{array}$ & $\begin{array}{l}1.3 \\
0.4\end{array}$ & $\begin{array}{r}223 \\
83\end{array}$ & $\begin{array}{r}219 \\
64\end{array}$ & $\begin{array}{l}1.6 \\
0.3\end{array}$ & $\begin{array}{l}1.0 \\
0.2\end{array}$ & $\begin{array}{r}171 \\
42\end{array}$ & $\begin{array}{r}490 \\
95\end{array}$ & \\
\hline
\end{tabular}

were initially 9.2 $\pm 7.1 \mathrm{KAU}$ and $395 \pm 207 \mathrm{mU} / \mathrm{ml}$, respectively, and after three weeks' therapy they increased to $12.7 \pm 7.9 \mathrm{KAU}$ and $461 \pm 177 \mathrm{mU} / \mathrm{ml}$ respectively. Both values increased after treatment, but the increase was insignificant in either case. On the basis of YAMAGUCHI's standard [16], the serum HSAP value was abnormally low in 7 patients $(23.3 \%)$ initially and in one patient $(3.3 \%)$ after treatment.

\section{Discussion}

In the diagnosis of IUGR, it is important to confirm the gestational age. Since the fetal growth during the first trimester follows a definite growth pattern, it would be better to ascertain the gestational age by routine ultrasonic measurements of either CRL during 6 to 15 weeks' gestation or BPD during 10 to 19 weeks' gestation. There are several ultrasonic methods to estimate the fetal body weight using $\mathrm{BPD}, \mathrm{AC}$, total inrauterine volume 


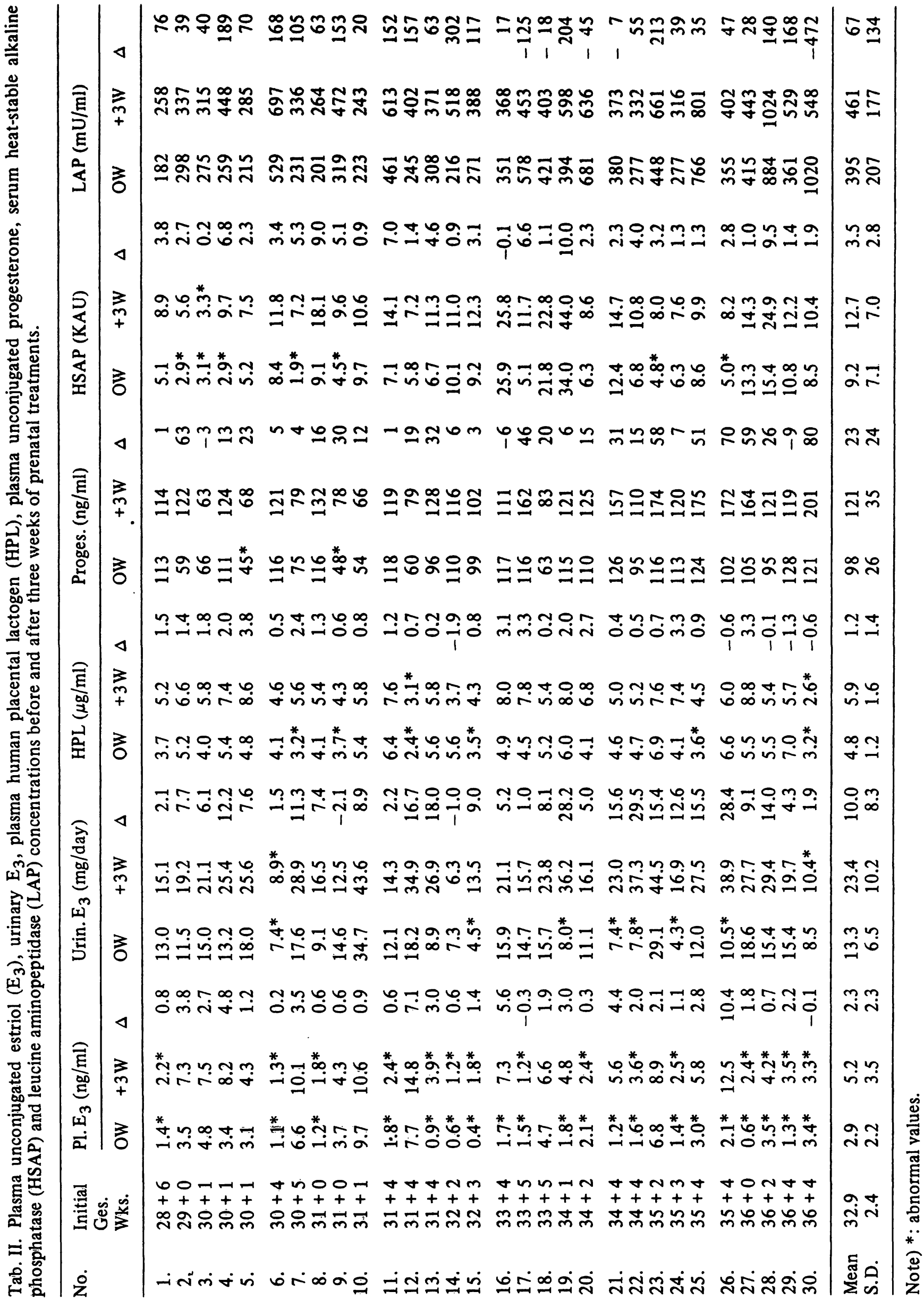


and so on. WARSOF et al. [14] reported that a logarithmic equation (equation $I$ in this study) with use of both BPD and AC had an absolute mean error of $228 \mathrm{~g}$ or $8.0 \%$. Later, SHEPARD et al. [8] stated that equation I and a new equation (equation II in this study) had standard deviations of $212 \mathrm{~g}$ and $219 \mathrm{~g}$ respectively, when the fetal body weight was less than $2,500 \mathrm{~g}$. In this study, the standard deviations of equation I and II were $178 \mathrm{~g}$ and $259 \mathrm{~g}$ respectively, for birth weight predictions. Correlation coefficients were 0.94 for both equations confirming that these equations for calculation of the estimated fetal body weight is useful in in the prenatal diagnosis of IUGR. Having taken these facts into consideration, we measured and corrected the gestational age of our IUGR patients in the first trimester, calculated the estimated fetal body weights from the BPD and $\mathrm{AC}$ measurements during the third trimester, and selected those patients whose estimated fetal body weights derived from at least two ultrasonic measurements separated by two weeks were less than the 10th percentile of the intrauterine growth curve of NISHIDA [4] for the Japanese.

In IUGR, there is fetal hypoplasia, with a decrease in both numbers of somatic cells and total DNA amounts in the tissue; there is also fetal malnutrition, with a decrease in both volume of somatic cells and protein/DNA ratio in the tissue. With the former, prenatal treatment is difficult; therefore, the underlying etiologies should be identified using antenatal diagnostic measures such as ultrasono- graphy. For nutritional disorders, daytime bedrest was indicated to enhance the placental bloodflow, and high protein diet was encouraged as well. Allylestrenol has been reported to increase urinary HCG, pregnandiol, HPL and estriol in pregnant women $[7,9,10]$, and it has been reported to have a placentotrophic, pregnancy-maintaining and weight-promoting activity in the fetus [7]. Allylestrenol does not have estrogenic, androgenic and anabolic effects as some of the gestagens $[1,3$, 15]. Indeed, the administration of allylestrenol was followed by significant increases of estriol, HPL and progesterone in this study, although some of those increments would be attributed to the increase in gestational age. An attempt to promote fetal weight without consideration of the functional status of the placenta is detrimental for the fetus, since this eventually increases the fetal volume/oxygen supply ratio. However, following allylestrenol treatment, the fetal/placental weight ratio was considerably smaller than that of normal pregnancy. Other important matters in the prenatal management IUGR would be:

1. to properly evaluate the fetal wellbeing and the intrauterine environment,

2. to determine the exact time of delivery while preventing both intrauterine fetal death and iatrogenic prematurity, and

3. to provide an efficient perinatal care to the newborn infant.

\section{Summary}

Prenatal treatment consisting of daytime bedrest, high protein diet and oral administration of allylestrenol was assessed in a prospective study of 30 patients with IUGR infants whose ultrasonically estimated body weight was less than the 10 th percentile. In these pregnancies, the gestational age was confirmed in the first trimester, and the fetal weight was estimated from the BPD and AC measurements in the third trimester (Fig. 1). Following treatment, ultrasonic and biochemical determinations were performed. As results:
1. The estimated fetal weight of $1,362 \mathrm{~g}$ at $32.9 \mathrm{~g}$ gestational weeks increased to $2,678 \mathrm{~g}$ at 39.2 weeks on average. The average weekly weight gain was significantly higher than the standard, and 16 cases $(53.3 \%)$ were more than 10th percentile at birth (Tab. I, Fig. 2).

2. A significant correlation $(r=0.94)$ between the estimated fetal weight and the birth weight was found.

3. Following prenatal treatments, maternal plasma and urinary estriol, pläsma HPL and progesterone increased significantly (Tab. II).

Keywords: Allylestrenol, estriol, fetus, HPL, HSAP, intrauterine growth retardation, LAP, progesterone, ultrasonographic measurement. 


\section{Zusammenfassung}

Pränatale Diagnose und Therapie der intrauterinen Wachstumsretardierung

In einer prosepektiven Studie untersuchten wir die Wirksamkeit einer pränatalen Behandlung der intrauterinen Wachstumsretardierung bei 30 Patientinnen. Die Therapie bestand in Verordnung von Bettruhe, proteinreicher Diat und oraler Gabe von Allylöstrenol. Das durch Ultraschall geschätzte Gewicht der Feten lag unterhalb der 10erPerzentile. Wir bestimmten das Schwangerschaftsalter im 1. Trimester und ermittelten im 3. Trimester das fetale Gewicht durch Messung des biparietalen Durchmessers und abdominellen Bauchumfangs (Fig. 1). Nach der Therapie führten wir ultrasonographische und biochemische Kontrollen durch. Unsere Ergebnisse:
1. Während das Gewicht in der 32,9. Schwangerschaftswoche bei durchschnittlich $1362 \mathrm{~g}$ lag, stieg es bis zur 39,2 . Woche auf ein mittleres Gewicht von $2678 \mathrm{~g}$ an. Die durchschnittliche Gewichtszunahme pro Woche war signifikant höher als der Standardwert. 16 Kinder $(53,3 \%)$ lagen mit ihrem Gewicht zum Zeitpunkt der Geburt oberhalb der 10-er-Perzentile (Tab. I, Fig. 2).

2. Die Korrelation zwischen geschätztem und tatsächlichen Geburtsgewicht war signifikant $(r=0,94)$.

3. Nach der pränatalen Therapie zeigten die Östriolwerte im Plasma und Urin sowie die HPL- und Progesteronwerte im Plasma einen signifikanten Anstieg (Tab. II).

Schlüsselwörter: Allylöstrenol, Fetus, hitzestabile AP, HPL, intrauterine Wachstumsretardierung, LAP, Östriol, Progesteron, Ultraschallmessung.

\section{Résumé}

Diagnostic prénatal et traitements du retard de croissance intra-utérine

Dans une étude prospective protant sur 30 patientes avec un enfant ayant un RCIU dont le poids corporel estimé par ultrasons est inférieur au dixième percentile, les thérapeutiques prénatales, à savoir le repos au lit quotidien, le régime hyperprotidique et la prise orale d'allylestrenol, ont été mises à l'épreuve. Pour ces grossesses, l'âge gestationnel a été confirmé au cours du premier trimestre et le poids fœtal estimé au cours du troisième trimestre par détermination du Bip et de l'abdomen (Fig. 1). A la suite des mesures thérapeutiques, ont été réalisés des examens biochmimiques et échographiques. Les résultats sont les suivants:
1. Le poids fœtal estimé de $1362 \mathrm{~g}$ à 32,9 semaines de gestation s'est accru jesqu'à $2678 \mathrm{~g}$ à 39,2 semaines en moyenne. Le gain pondéral hebdomadaire moyen a été plus élevé de façon significative que normalement et 16 cas $(53,3 \%)$ ont eu un poids supérieur au 10 ème percentile à la naissance (Tab. I, Fig. 2).

2. Une corrélation significative $(r=0,94)$ a été mise en évidence entre l'estimation du poids fœtal et le poids de naissance.

3. A la suite des thérapeutiques prénatales, l'estriol maternal plasmatique et urinaire, l'HPL plasmatique et la progestérone ont augmenté de façon significative (Tab.II).

Mots-clés: Allylestrenol, déterminations échographiques, estriol, fetus, HPL, progestérone, retard de croissance intrautérin.

\section{Bibliography}

[1] AYDAR, C. K., R. B. GREENBLATT: Clinical observations on a new progestational agent "Allylestrenol". Acta Endocr. 38 (1961) 419

[2] BEISCHER, N. A., J. B. BROWN, M. A. SMITH, L. TOWNSEND: Studies in prolonged pregnancy. II. Clinical results and urinary estriol excretion in prolonged pregnancy. Amer. J. Obstet. Gynec. 103 (1969) 483

[3] BORGLIN, N. E.: Clinical evaluation of the progestational effect of allylestrenol. Acta Endocri. 51 (1960) 929

[4] NISHIDA, H.: Intrauterine growth charts of Japanes. I. Composition of the intrauterine growth charts. Acta neonat. Jap. 14 (1978) 557

[5] ROBINSON, H. P.: Sonar measurement of fetal crown-rump length as means of assessing maturity in first trimester of pregnancy. Brit. Med. J. 4 (1973) 28

[6] SABBAGHA, R. E., F. B. BARTON, B. A. BARTON: Sonar bi-parietal diameter. I. Analysis of percentile growth difference in two normal populations using same methodology. Amer. J. Obstet. Gynec. 126 (1976) 479

[7] SAS, M., V. RAPCSÁK, I. OROJON: Untersuchungen über die Wirksamkeit des Allylöstrenol in der Behandlung wiederholter Abortus. Zbl. Gynaek. 87 (1965) 1544

[8] SHEPARD, M. J., V. A. RICHARDS, R. L. BERKOWITZ, S. L. WARSOF, J. C. HOBBINS: An evaluation of two equations for predicting fetal weight by ultrasound. Amer. J. Obstet. Gynec. 142 (1982) 47

[9] SPONA, J., E. MÜLLER-TYL, S. LEODOLTER: Einfluss von Allylöstrenol auf HPL-Serumspiegel bei Risikoschwangerschaften. Z. Geburth. Perinat. 180 (1976) 356

[10] SZONTÁGH, F. E., M. SAS, A. TRAuB, L. KOVÁCS, Á. BARDÓCZY, Z. SZEREDAY: The influence of different norsteroids on the hormone excretion and on the histomorphologic pattern in the trophoblast in early pregnancy. Gynaecologia 156 (1963) 369 
[11] TAMURA, R. K., R. E. SABBAGHA: Percentile ranks of sonar fetal abdominal circumference measurements. Amer. J. Obstet. Gynec. 138 (1980) 475

[12] TULCHINSKY, D., D. M. OKADA: Hormones in human pregnancy. IV. Plasma progesterone. Amer. J. Obstet. Gynec. 121 (1975) 293

[13] VARMA, K., S. G. DRISCOLL, K. JR. EMERSON, H. A. SCLENKOW: Clinical and pathologic evaluation of serum immunoreactive human placental lactogen (IR-HPL) in abnormal pregnancy. Obstet. and Gynec. 38 (1971) 487

[14] WARSOF, S. L., P. GOHARI, R. L. BERKOWITZ, J. C. HOBBINS: The estimation of fetal weight by computer-assisted analysis. Amer. J. Obstet. Gynec. 128 (1977) 881
[15] WU, D. H.: Gestational effect of allylestrenol. Endocrinol. Japon. 9 (1:962) 187

[16] YAMAGUCHI, R., I. YOSHIDA, S. YAZAKI: Placental function test by serum heat-stable alkaline phosphatase in pregnant women. Nippon Sanka Fujinka Gakkai Zasshi 20 (1968) 567

Received December 27, 1982: Accepted March 23, 1983.

Dr. Tsuyoshi Kaneoka

Fukuoka University School of Medicine 7-45-1 Nanakuma, Jyonanku, Fukuoka 814-01, Japan 